\title{
Lithium effects on stem cells - advances in stem cell application in clinical medicine.
}

\author{
Vincent S Gallicchio* \\ Departments of Biological Sciences, Microbiology, and Public Health Sciences, Clemson University, USA
}

\begin{abstract}
Lithium (Li) salts have been widely used in psychiatry as mood stabilizing agents for 66 years. Li is found in variable amounts in foods, especially grains, vegetables, and in various geographical areas. Additionally drinking water provides a significant source of the element. Dietary intake in humans depends on location, type of foods consumed, and fluid intake. Traces of $\mathrm{Li}$ have been detected in human organs and tissues, leading speculation that the element is responsible for specific functions in the human body. It was not until the 20th century that studies performed in the 1970's and 1990's, primarily in chickens, cows, goats, and rats, maintained on Li-deficient diets demonstrated higher mortality, and altered reproductive and behavioral abnormalities. Such deficiencies have not been detected in humans; however, studies performed on populations living in areas with lower Li levels in water supplies have been associated with higher rates of suicides, homicides, and the arrest rates for drug abuse and other crimes. Thus, $\mathrm{Li}$ appears to play a significant role in early fetal development evidenced by high $\mathrm{Li}$ levels during the early gestational period and perhaps social behavior. Biochemically, the mechanism of $\mathrm{Li}$ action involves multi-factor and interconnected pathways with enzymes, hormones, vitamins, transcription, and growth and transforming factors involved. This body of evidence now appears sufficient to label $\mathrm{Li}$ as an essential element with the recommended RDA for a $70 \mathrm{~kg}$ adult of $1 \mathrm{mg} / \mathrm{day}$. Of extreme importance for the future is the increasing clinical data indicating Li can be used effectively for the treatment of acute brain injuries, e.g., ischemia and chronic neurodegenerative diseases such as Alzheimer's disease, Parkinson's disease, Tauopathies, and Huntington's disease. This conclusion is based upon evidence showing $L i$ as important in neurogenesis, neuronal repair, as well as protecting neurons from neurotoxicity.
\end{abstract}

Keywords: Lithium, Stem cells, Medical uses.

Accepted on January 2, 2018

\section{Introduction and Overview}

Lithium (Li) salts have been widely used in psychiatry as mood stabilizing agents for 66 years. $\mathrm{Li}$ is found in variable amounts in foods, especially grains, vegetables, and in various geographical areas. Additionally, drinking water provides a significant source of the element in certain geographical areas as well. Dietary intake in humans depends on location, type of foods consumed, and fluid intake. Traces of $\mathrm{Li}$ have been detected in human organs and tissues, leading speculation that the element is responsible for specific functions in the human body. It was not until the 20th century that studies performed in the 1970's and 1990's, primarily in chickens, cows, goats, and rats, maintained on $\mathrm{Li}$ deficient diets demonstrated higher mortality, and altered reproductive and behavioral abnormalities. Such deficiencies have not been detected in humans; however, studies performed on populations living in areas with lower Li levels in water supplies have been associated with higher rates of suicides, homicides, and the arrest rates for drug abuse and other crimes. Thus, Li appears to play a significant role in early fetal development evidenced by high Li levels during the early gestational period and perhaps social behavior. Biochemically, the mechanism of $\mathrm{Li}$ action involves multi factor and interconnected pathways with enzymes, hormones, vitamins, transcription, and growth and transforming factors involved. This body of evidence now appears sufficient to label $\mathrm{Li}$ as an essential element with the recommended RDA for a $70 \mathrm{~kg}$ adult of $1 \mathrm{mg} /$ day. Of extreme importance for the future is the increasing clinical data indicating Li can be used effectively for the treatment of acute brain injuries e.g., ischemia and chronic neurodegenerative diseases such as Alzheimer's disease, Parkinson's disease, Tauopathies and Huntington's disease. This conclusion is based upon evidence recently reviewed [1] showing $\mathrm{Li}$ as important in neurogenesis, neuronal repair, as well as protecting neurons from neurotoxicity. Finally, Li influences stem cells, both neuronal and marrow derived, and from multiple organs and tissues. Thus, additional therapeutic implications for this element in clinical medicine to treat disorders associated with the faulty production of blood and nerve cells or as a tool to enhance blood stem cell mobilization for transplantation warrants further research.

Lithium (from Greek lithos 'stone') is a chemical element with symbol $\mathrm{Li}$ and atomic number 3 . It is a soft, silver white metal belonging to the alkali metal group of chemical elements. Under standard conditions it is the lightest metal and the least dense solid element. Like all alkali metals, lithium is highly reactive and flammable. For this reason, it is typically stored in mineral oil. When exposed, lithium exhibits a metallic luster, but contact with moist air corrodes the surface quickly to a dull silvery gray and then black tarnish. Because of its high 
reactivity lithium never occurs freely in nature and instead, only appears in compounds, which are usually ionic. Lithium occurs in a number of pegmatite minerals, but due to its solubility as an ion is present in ocean water and is commonly obtained from brines and clay. On a commercial scale, lithium is isolated electrolytically from a mixture of lithium chloride and potassium chloride.

Lithium and its compounds have several industrial applications, including use in the manufacture of heat resistant glass and ceramics, high strength-to-weight alloys used in aircraft, lithium batteries, and lithium ion batteries. These commercial uses consume more than half of lithium annual production.

Trace amounts of lithium are present in all organisms. The element serves no apparent vital biological function under normal physiological conditions, unless it is deprived or eliminated, since animals and plants survive in good health without it; however, its role in avian and mammalian reproduction has been documented as previously noted. In the absence of lithium when tested in a number of vertebrate species (fowl, cows and goats) all animals tested showed an increased in spontaneous abortions among animals tested. Thus, the implied link is that lithium is an essential component required for normal gestation during pregnancy. Clinically in humans, the lithium ion $[\mathrm{Li}+]$ administered as one of the several available lithium salts have all proved to be useful as a mood stabilizing drug in the treatment of bipolar disorder due to the neurological effects of the ion in the human body.

In nature, lithium is found in trace amounts in numerous plants, plankton and invertebrates at concentrations of 69 to 5,760 parts per billion (ppb). In vertebrates the concentration is slightly lower, and nearly all vertebrate tissue and body fluids have been found to contain lithium ranging from 21 to $763 \mathrm{ppb}$ [2]. Marine organisms tend to bio accumulate lithium more than terrestrial ones [3]. It is not known whether lithium has a physiological role in any of these organisms [3], but nutritional studies as stated above in birds and mammals have indicated its importance to health, leading to a suggestion that it be classed as an essential trace element with an RDA of $1 \mathrm{mg} /$ day [4]. Observational studies in Japan, reported in 2011, suggested that naturally occurring lithium in drinking water might develop Ebstein's cardiac anomaly in infants born to women who take lithium during the first trimester of pregnancy [5]. The most exciting results for lithium are its reported nonpsychiatric medical effects that are centered on the ion's ability to influence the proliferation of stem cells first identified from hematopoietic tissues [6,7]. This effect has now been demonstrated to also take place in the regeneration of neurological tissues and stem cells derived from other organs and tissues, making the use of lithium to treat spinal cord and other system and organ injuries through a mechanism that involves enhancing stem cell regeneration of damaged neurons very promising for the future of clinical medicine [8].

\section{Lithium and Cell Biology}

Lithium is still the gold standard treatment for bipolar disordermanic depression, a severe mental illness. Lithium, which has a well-established safety profile, has been FDA approved and used to treat bipolar disorders for more than 60 years [9]. It is an effective treatment for mania and is also used as prophylactic therapy to prevent the recurrent manic and depressive episodes that characterize bipolar disorder [10]. A large body of evidence suggests that inflammation plays a role in the pathogenesis of bipolar disorder and that mood stabilizers exhibit anti-inflammatory properties. Pretreatment with lithium $10 \mathrm{mM}$ (but not $1 \mathrm{mM}$ ) significantly reduced LPSinduced secretion of TNF, IL-1 $\alpha$, prostaglandin-E2 and nitric oxide in rat primary glia cells [10]. In addition, lithium significantly reduced the expression of cyclooxygenase- 2 and inducible nitric oxide synthase, findings suggesting that lithium exhibits a potent anti-inflammatory effect [11]. Further evidence from our laboratory suggested potent anti-cancerous and apoptosis modulating activities by lithium on HL-60 promyelocytic leukemia cells and hematopoiesis [12-17]. This is compelling evidence that supports the notion that treatment with lithium may elicit strong anti-inflammatory effects in inflammation and in cancerous cells. Hence, a promising chemotherapeutic direction has emerged from these observations based upon lithium's initial clinical use in psychiatry and a monovalent cationic element.

Pioneering studies demonstrated lithium directly inhibits glycogen synthase kinase-3 beta (GSK-3 $\beta$ ) activity $[18,19]$. This enzyme has been further established as a crucial target for lithium's cellular effects [20,21]. GSK-3 $\beta$, consisting of a and $\mathrm{b}$ isoforms, is a serine/threonine kinase that regulates diverse cellular and neurophysiological processes and has been implicated as one of the main causative agents responsible for the development of Alzheimer's disease. Lithium competes with magnesium to directly inhibit GSK-3 $\beta$ by binding to the active site of the enzyme and limiting magnesium's catalytic activity [22]. Lithium also indirectly inhibits GSK-3 $\beta$ activity by enhancing phosphorylation of GSK-3 $\beta$ at Ser21 and GSK-3 $\beta$ at Ser9 via activation of phosphatidylinositol 3-kinase [PI3-K]/Akt, protein kinase A and protein kinase C $[22,23]$. In addition, lithium has been shown to increase the activities of two transcription factors, activator protein-1 [AP-1] and cyclicAMP response element binding protein [CREB], both in vivo and in vitro [24,25]. Lithium also activates the mitogenactivated protein [MAP] kinase pathway [26]. These metabolic pathway intermediates play a crucial role in regulating apoptosis, cytokine production and differentiation in HL-60 cells [27].

Lithium is a potent inhibitor of GSK-3 $\beta$. GSK-3 $\beta$ inhibition has been demonstrated to have anti-inflammatory effects, as shown by reduced TNF- $\alpha$ production via attenuated activation of NF-k $\beta$ and JNK signaling cascades [28] and induction of the anti-inflammatory cytokine, IL-10 [29]. GSK-3 $\beta$ and NF-k $\beta$ play a central role in cancer progression, and regulation of the factors by lithium may prove to be important in cancer treatment. In light of these findings, the effects of lithium on 
Citation: Gallicchio VS. Lithium effects on stem cells - advances in stem cell application in clinical medicine. Adv Cell Sci Tissue Cul 2018;2(1):14-24.

expression of apoptosis-related genes and inflammation associated cytokines have been studied.

\section{Lithium and Cancer}

Although lithium was first tested for its use in the treatment of mania, during recent years attention has been focused on its anti-cancer potentials as well. With lithium use in psychiatry for $60+$ years, there is no clinical evidence associating lithium use in psychiatric patients with the formation of any neoplastic blood cell dyscrasias. It is now well accepted that in laboratory studies, lithium concentrations of $10 \mathrm{mM}$ and above inhibit cell growth of HL-60 promyelocytic leukemia cells [11-17,21,22]. Although $10 \mathrm{mM} \mathrm{LiCl}$ induces apoptosis in HL-60 cells, a small fraction of the cell population remains viable, survives the cytotoxic effects of lithium and exhibits an altered gene expression pattern when sub-cultured for lengthy periods [4]. The resistance acquired by these HL- 60 cells requires further evaluation as results from these studies will be of importance not only in psychiatry but in the proposed use of lithium as a chemotherapeutic agent. Results from our laboratory demonstrated lithium seems to be selectively cytotoxic to cancerous cells while sparing non-cancerous cells, a phenomenon important in noteworthy that although $\mathrm{Li}$ is associated with an increased drug as therapy for bipolar and manic depressive, this increase in neutrophils is reversible and has not been associated with the development of any leukemia.

Evidence from our laboratory and others indicated lithium resistance acquired by HL-60 cells and its anti-cancerous effects include a mechanism of action involving the induction of apoptosis accompanied by modulation of apoptosis related genes [30,31]. HL-60 cells express two important apoptosis related genes: bax, a pro-apoptotic gene, and bcl-2, an antiapoptotic gene. Lithium appears to induce apoptosis in HL-60 cells by increasing the bax/bcl-2 ratio, and when this ratio is reversed, HL-60 cells acquire resistance to lithium cytotoxicity. When lithium concentrations were increased in the lithium resistant HL-60 cells, bcl-2 expression decreased and cell death increased.

In cells, the increase in lithium concentration to $15 \mathrm{mM}$ was accompanied by DNA fragmentation and a decrease in cell viability. Although treatment of HL-60 cells with $15 \mathrm{mM}$ lithium resulted in DNA fragmentation, this phenomenon was not observed in the lithium resistant sub-clones [HL-60LiR cells]. Expression of other apoptosis related gene products such as p53 and $\mathrm{Rb}$ increased proportionally with increased lithium dosage. The tumor suppressor protein, $\mathrm{Rb}$, was significantly elevated especially after treatment of HL-60 cells with $20 \mathrm{mM}$ lithium. Expression levels of $\mathrm{Rb}$ were generally lower in the HL-60LiR cells, an observation that could possibly be linked to the lithium resistance phenomenon acquired by these subclones. The p53 protein, like $\mathrm{Rb}$, is a nuclear protein that is postulated to arrest growth in the G1 phase of the cell cycle in response to a death signal or aberrant DNA activity [32]. When HL-60 cells were treated with higher doses of lithium, its expression levels increased albeit barely detectable in these cell lines, and as a result, very little could be deduced from these observations.
Since the successful growth and survival of leukemic cells is partly due to their ability to respond to local paracrine growth factors and inhibitors, the levels of several growth regulating or inflammation related cytokines were carefully investigated. TNF- $\alpha$, IL-6, IL-2 and IL-10 are several of the cytokines produced by HL-60 and HL-60LiR cells following detection using ELISA assays after $24 \mathrm{hrs}$ of lithium treatment. Both IL-6 and TNF- $\alpha$ have been shown to mediate apoptotic as well as necrotic forms of cell death in leukemic cell lines. Furthermore, it was previously reported that lithium considerably potentiates the antitumor activity of TNF- $\alpha$ and IL-6 both in vivo and in vitro. Lithium chloride has also been demonstrated to considerably increase the amount of TNF- $\alpha$ and IL-6 in murine skin [33]. Treatment of HL-60 and HL-60 LiR cells with lithium resulted in a dose-dependent increase of TNF- $\alpha$ and IL- 6 production. The increase in TNF- $\alpha$ and IL- 6 levels in both cells correlated with increase in lithium dependent growth inhibition and cell death induction. Production of these cytokines was comparatively higher when cells were treated with $20 \mathrm{mM}$ lithium, suggesting that induction of cell death in HL-60 and HL-60LiR cells might be achieved via modulation of TNF- $\alpha$ and IL- 6 production. This report supports findings from previous studies, which proposed that lithium and TNF- $\alpha$ work in synergy when inhibiting growth of HL-60 cells. Hence, the lithium induced cell death may be a direct consequence or indirect stimulation of TNF $\alpha$.

Production of IL-2 and IL-10 in HL-60 cells is generally decreased by lithium in a dose dependent fashion. Both IL-2 and IL-10 are reported as potent growth promoting cytokines in HL-60 cell lines. Thus, IL-2 and IL-10 represent potent positive progression signals for the growth and differentiation of their cellular targets, especially, leukemic subtypes [34]. Survival of cancer cells depends on the balance between growth promoting cytokines and growth inhibitors; hence, when growth factors, like IL-2 and IL-10 are low and growth inhibitors, such as TNF- $\alpha$ and IL- 6 are elevated, cells are destined to die. Surviving HL-60 LiR cells may, therefore, be evading the process of cell death by decreasing levels of TNF$\alpha$ and IL- 6 while promoting IL- 2 and IL-10 production. Thus, the survival patterns seen in HL-60 LiR cells might be a result of increased IL-2 and IL-10 levels with concomitant reduction in the production of TNF- $\alpha$ and IL-6. Induction of cell death by lithium in HL-60 cells may involve stimulation of IL-6 and TNF- $\alpha$ secretion and concomitant inhibition of IL- 2 and IL-10 production. Taken together, these results suggest lithium may be inducing cell death in HL-60 and HL-60LiR cells by modulating cytokine production and expression of apoptosis related genes. The use of lithium in cancer management would improve treatment accessibility and reach a wider patient community, even those in developing countries, due to its affordability, stability and extensive distribution.

Lithium has been used clinically to treat granulocytopenia, chemotherapy induced neutropenia, aplastic anemia, radiation induced neutropenia, childhood neutropenia, autoimmune neutropenia, agranulocytosis associated with hyperthyroidism, hepatitis associated agranulocytosis, and clozapine induced neutropenia. All are dyscrasias with a known etiology centered 
on faulty or inadequate stem cells. In most of these cases tested, lithium had beneficial effects, although it paradoxically increased neutropenia duration during cyclic neutropenia by amplifying granulocytic oscillations, suggesting that lithium may have complex effects on cyclic phenomena involving suppressor activities and interactions between different populations of white blood cells and potentially stem cells. In some hematopoietic disorders, lithium can be dramatically effective. For example, lithium enhances peripheral blood neutrophil, serum CSA levels, and other symptoms in Felty's syndrome, an autoimmune condition with rheumatoid arthritis, leukopenia, and splenomegaly. In some cases, lithium alone cannot reverse this condition and must be given alongside high dose corticosteroids. However, in many cases, a single course of lithium carbonate restores neutrophil counts to normal. Lithium does not affect all granulocytes to the same extent. In the presence of low lithium concentrations its effect on producing myeloid colonies from normal patients and those with overt acute myelomonocytic leukemia [AMML] are not significant. In myeloid colonies from normal patients, lithium increased the sizes of colonies by 1.5-2.0 times and cell cycle analysis showed a significant increase in percentage of cells in S, G2, and M phases. However, lithium had no effect on cell colonies grown from patients with acute myelomonocytic leukemia (Figure 1).

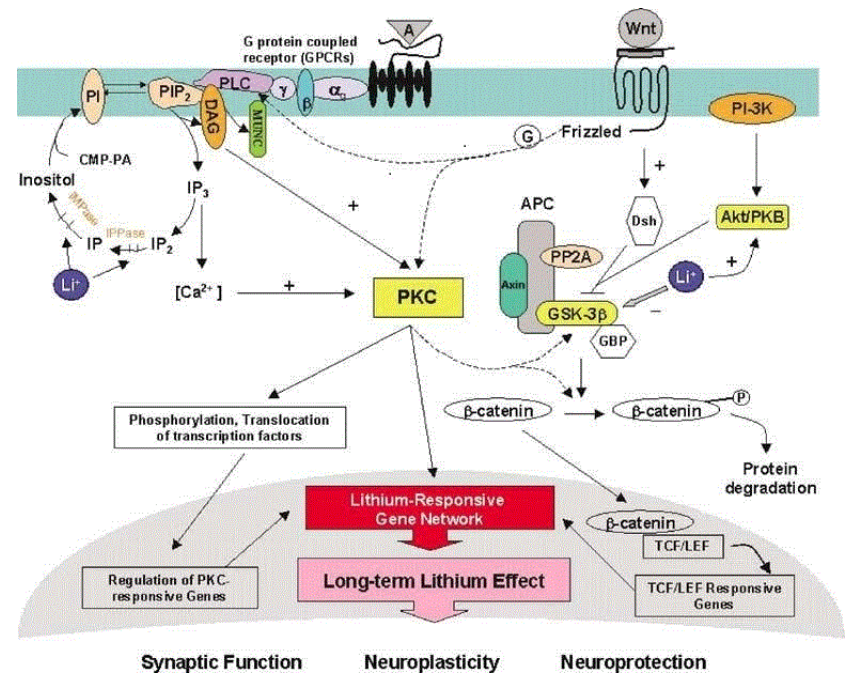

Figure 1: Signal transduction mechanism for lithium action.

\section{Lithium and Animal Cells}

Lithium has been used in humans as a drug for depression and other psychiatric problems for several decades. In addition, lithium has also been used to treat different diseases in animals. In the hopes of one day using lithium to treat hemorrhagic fevers in humans such as Ebola, case studies were reviewed on hematopoietic properties of lithium in animals with a concentrated focus on blood disorders and cellular level mechanisms of lithium on stem cells in animals.

Lithium has been used in animals to treat gray collie dogs that develop cyclic hematopoiesis [35]. Cyclic hematopoiesis causes cycling of hematopoietic stem cells, which causes not only thrombocytopenia but also neutropenia. This disease leads to numerous infections and possible death. The current therapy options are bone marrow transplantation or lithium carbonate as a drug. In one study [35] lithium carbonate tablets were given orally twice daily with endotoxin injections. Results showed cycles of neutropenia stopped, neutrophil counts stabilized, as did monocytes and platelet counts, and hematocrit levels increased. Once lithium was discontinued all cycling returned to its previous state. The one side effect noted was an anemia when lithium carbonate is used with endotoxin. This effect is likely due to the inflammatory nature of endotoxin in addition to lithium's ability to induce leukocytosis rather than erythropoiesis. Overall Li treatment has a positive effect on the proliferation of hematopoietic stem cells and as such demonstrates promising results that with more tests could prove to be very useful tool to improve clinical transplantation in human medicine.

Overall these clinical and experimental findings peaked interest to explore how or to what therapeutic effect lithium could have on other blood disorders that centered on the development of thrombocytopenia. Many studies have been performed where thrombocytopenia [a marked reduction of platelets in the peripheral blood] was induced by another drug. Lithium was then used to counteract these inhibitory effects on platelets. One such study, [36] where lithium carbonate was used to counteract the effects of carboplatin-induced thrombocytopenia in dogs, eighteen dogs used in the study that generated the following results. All dogs that received lithium reached a systemic lithium blood level of 0.5-1.5 mmol/L, which was the target level and mimics levels used clinically to treat manic depressive and bipolar patients. Thrombocytopoiesis was increased in all dogs receiving lithium. The authors concluded lithium-stimulated thrombocytopoiesis, but when given with carboplatin and cephalexin, lithium did not prevent thrombocytopenia, but it did induced thrombocytopoiesis.

Another study investigated the use of lithium carbonate to treat myelosuppression in dogs [37]. In this study the levels of lithium used were higher than recommended for use clinically in humans, thus the researchers concentrated on the toxic levels of lithium when used to counteract drug effects. This study used 4 beagles and over all found that lithium had many toxic side effects when dosed too high. Lithium carbonate was used with lomustine as the two counter acting drugs. Lomustine is a cytotoxic drug that is given to treat neoplasms in cancer patients, with side effects being neutropenia and when given an acute-doses and induces thrombocytopenia when given at chronic doses. The researchers also found lithium carbonate combined with lomustine at too high concentrations caused seizures in dogs. The dogs treated with both drugs also had ptyalism along with lethargy, inappetence, hypodipsia, vomiting, diarrhea, and weight loss. However, once the treatment was stopped the dogs returned to normal despite showing signs of neutropenia. There were four limitations to the conclusion:

[a] There were only two dogs for each treatment group; therefore the study groups were small in numbers. 
Citation: Gallicchio VS. Lithium effects on stem cells - advances in stem cell application in clinical medicine. Adv Cell Sci Tissue Cul 2018;2(1):14-24.

[b] They did not use consistent doses of lithium within a therapeutic level, because the optimal concentration for hematopoietic stimulation is not yet known in dogs.

[c] Lithium was administered daily instead of 24 hours after every lomustine treatment. It was administered daily to study the effects of toxicity.

[d] Cephalexin, an antibiotic was given to prevent sepsis, may have interfered with the lithium medication. Overall this study showed that while there were positive therapeutic effects associated with lithium use, as all drugs are toxic at a certain dose and when clinical trials are used the dosage needs to be studied closely.

A review of estrogen induced myelotoxicity in dogs showed promising results when using lithium as therapy [38]. Estrogens or synthetic estrogenic compounds have been used in female dogs for the treatment of insufficient mating practices, hormonal urinary incontinence, estrus induction, or pseudo pregnancy and in male dogs for prostatic hypertrophy, perianal adenoma or testicular neoplasms. The use of estrogen can cause myelotoxicity. The side effects occur in three stages:

Stage 1: A brief increase in platelet numbers followed by a severe thrombocytopenia;

Stage 2: Bone marrow granulocyte hyperplasia with neutrophilia,

Stage 3: Marrow recovery or marrow aplasia. Treatment with lithium has been used in dogs with EIM [estrogen induced myelotoxicity] and in each case, the treated animals made a complete recovery. Therefore, the effect of lithium to stimulate the proliferation of pluripotent stem cells demonstrated the return of erythrocyte, leukocyte, and platelet to normal values.

A study on a female pincer dog that developed cysts on its ovaries due to over productions of estrogen, led to induction of neutropenia and bone marrow aplasia. These conditions were treated effectively with lithium carbonate for 88 days with the results demonstrating the animal was cured of all side effects [39]. The dog showed a weekly increase in blood cell numbers, resolving the leukopenia by the fourth week of treatment. The anemia had become regenerative and the neutrophil count was within the reference range. By the seventh week, the white blood cell count and packed cell volume were normal. While a discreet anemia could still be observed, the dog was clinically healthy. This study supports the successful use of and treatment of lithium carbonate for canine bone marrow aplasia.

After reviewing these articles the focus then was centered on the cellular effects of lithium. Two articles provide important information-in one study [40] it was reported lithium has inhibitory effects on GSK $3 \beta$ activity and in the WNT signaling process. These observations were found to be similar when tested using cardiac and skeletal muscle cells in mice. Another study [41] also demonstrated lithium affects GSK3 $\beta$ and the WNT signaling pathway when tested in chicken cells. The WNT signaling pathway is important for neuronal development and axis formation, in addition to playing an important role in the formation of cancer. Lithium mimics the
WNT pathway, which in turns inhibits the GSK3 $\beta$ pathway and leads to increased levels of $\beta$-catenin. Knowing that lithium works mechanistically through these signal transduction pathways provides important knowledge for future development exploring lithium as a method to treat neural diseases and cancer.

Further research should focus to determine the correct and safe dosage levels of lithium to treat dogs and cats clinically, which could help prevent the toxicity issues that were identified in the discussion above. Additional research should be performed to determine if lithium may be clinically effective in minimizing and/preventing virally induced blood disorders such as hemorrhagic fevers, which would reconnect the research back to the starting point of Ebola. Finally, the actual mechanism of lithium at the cellular level needs to be further investigated, not only on its mechanism of action on stem cells, but also on regular living cells in the body.

\section{Lithium and Hematopoietic Stem Cells}

In the treatment of manic depression and bipolar illness in humans, lithium remains the gold standard. In these cohorts of patients who receive lithium therapy over time a side effect was observed patients develop a leukocytosis that was manifested primarily as neutrophilia that was sustained, dose dependent, and importantly at the same time, not pathogenic. The idea that a monovalent cation could be responsible for this response was intriguing. Studies conducted in our laboratory were designed to determine if lithium, when administered in a dose response manner to normal mice would influence the proliferation of hematopoietic stem cells as a plausible explanation for the increase in neutrophilia seen clinically. The results of these studies showed at doses used clinically to treat bipolar and manic depressive patients; lithium increased the number of pluripotential hematopoietic stem cells [CFU-S] in a dose dependent manner when tested in normal mice. These results demonstrated for the first time that a monovalent cation is capable of influencing the proliferation of pluripotential stem cells thus increasing their number [6]. The topic of lithium and blood effects has been the subject of many reviews and textbooks. Author refer the reader to the following references where this topic is discussed more extensively [7,42-51].

Because lithium is an effective agent capable of stimulating the proliferation and thus the production of hematopoietic stem cells, whether lithium is combined with bone marrow cells in an in vitro tissue culture environment such as in cell culture or in a long-term bone marrow culture system or following administration in vivo, it was then hypothesized lithium could play an important role clinically in human hematopoietic stem cell transplantation. This idea was proven efficacious after preclinical studies performed in mice demonstrated that pretreating donor animals with lithium at concentrations previously demonstrated to optimally increase pluripotential hematopoietic stem cells in vivo [6], when lithium was used to pre-treat donor animals, lithium stimulated an enhanced recovery of all hematopoietic elements [red cells, white cells and platelets] and did so by recovering at a more rapid response when compared to the rates of hematopoietic 
reconstitution in animals transplanted with marrow cells from mice receiving only saline as a control [50]. With this effect of lithium the question was then posed anatomically speaking where in bone marrow was this lithium effect manifested? Because it was known that lineages of hematopoietic stem cells were located in specific areas within the bone marrow cavity, it was important to know where anatomically this stem cell proliferation was located. Performing additional studies in mice demonstrated that the populations of pluripotential stem cells most influenced by lithium were located in the endosteal areas of bone marrow rather than the axial area of distribution within the marrow cavity [51]. These results pinpoint the specific area within hematopoietic tissue where the concentration of hematopoietic cells would be present in larger numbers, a finding important for the harvesting of stem cells for transplantation.

As stated previously, there is an interaction between lithium and the inhibitory enzyme GSK $3 \beta$. Recent studies have focused on the relationship between GSK3 $\beta$ and hematopoietic stem cell function and renewal. As stated previously in this review GSK3 $\beta$ involves action on intracellular functions through the WNT signaling system. There is evidence to show the ability of lithium to influence the proliferation of hematopoietic stem cells is mediated via lithium's influence on inhibiting GS3K $\beta$ action in mammalian cells [52]. Thus, the capacity of lithium to improve and make more effective clinical hematopoietic stem cell transplantation whether it is to either expand hematopoietic stem cells in vivo or in vitro is mediated by its inhibiting action on GSK3 $\beta$ [52].

\section{Lithium and Induced Pluripotential Stem Cells}

The science of stem cell biology has been dramatically advanced with the discovery of stem cells present not just from a variety of specific tissues, but importantly by the fact that somatic cells can be reprogrammed into induced pluripotential stem cells [iPSCs]. Initially the opportunity to produce iPSCs relied on the utility of either oncogenes or viral factors serving as vectors to induce cells to revert back to the stem cell stage; however, the efficiency of these agents to accomplish this task was poor at best, thus, the need arose to find new agents to promote iPSCs. Lithium has been shown to greatly enhance iPSCs production from both embryonic fibroblast and umbilical vein endothelial cells [53]. Lithium facilitated iPSCs production through enhancement of one or two of the defined transcription factors that are critical for the transformation of somatic cells to revert into iPSCs. These transcription factors [Oct4, Sox2, Klf4, c-Myc, Nanog and Lin28] have shown to be key to the reprogramming of somatic cells to iPSCs. Lithium has also been shown effectively to not just increase the expression of the transcription factor Nanog [40,41], but at the same time it also enhanced the expression of Nanog. Thus, this data opens the possibility of using lithium to engineer somatic cells to become iPSCs and by doing so may enhance the ability to improve stem cell based therapies.

Since there has been a relationship clinically in psychiatry with the use of lithium in the treatment of bipolar disorder manic depressive illness, the question was raised regarding whether inducing the formation of iPSCs from bipolar patients would improve our understanding of the events surrounding the etiology of this psychiatric condition and importantly would improve our understanding how lithium acts to function mechanistically in these patients could be further elucidated. A recent study reported that iPSCs generated from bipolar manic depressive patients showed altered transcription factors expression compared to controls [54]. Importantly the neurons that were produced from these patient derived iPSCs generated neurons that also differed from controls. The addition of lithium to the altered bipolar manic depressive derived iPSCs was reversed, thus implying lithium can reverse altered transcription factor expression and thus adds to growing knowledge of how lithium works mechanistically in humans expressing these psychiatric conditions. Again, lithium expresses utility in terms of how it can effectively modulate a number of different sources of stem cells, each with their own area of clinical utility in treating human disease.

\section{Lithium and Mesenchymal Stem Cells}

Because mesenchymal derived stem cells [MSCs] are multipotential cells that have been widely used as an alternative clinical therapeutic approach in cell based transplantation therapy, the use of MSCs must first focus on the requirement to procure as many of these stem cells as possible in order to meet the proper clinical threshold to produce an optimal clinical response in directed patients. In order to achieve this goal requires one to increase the production of these stem cells. To achieve this goal requires one to increase the cell replication [division] of these cells. Because this procedure needs to be performed in a tissue culture laboratory facility in order to optimize the production of these cells necessary to have a sufficient quantity to use clinically is referred to as in vitro clonal stem cell expansion. Therefore, the ability to increase the proliferative rate of MSCs it is a critical factor necessary to determine whether the use of MSCs in the clinical setting will achieve and/or surpass the therapeutic efficacy of standard therapeutic treatment for that specific clinical condition in which use of MSCs are being considered. In a recent study [55], it was demonstrated for the first time that lithium, reversibly promoted the proliferation of human bone marrow derived MSCs in vitro. MSCs co-cultured in the presence of $5 \mathrm{~mm}$ lithium demonstrated increase proliferation at an enhanced rate more rapidly than untreated cells and at the same time there was now information demonstrating that these induced cells expressed expansion without demonstrating signs of apoptosis. Lithium increased the proportion of MSCs in S phase as well as increasing the intracellular expression of cyclin D1, a known transcription factor.

\section{Lithium and Alzheimer's Disease}

Alzheimer's disease [AD] is most prominent when characterizing neurodegenerative diseases and as such is the most common form of dementia. It was named the 6th leading cause of death in America in 2015 [47]. An estimated 5.5 million Americans of all ages have Alzheimer's disease. Of the 
Citation: Gallicchio VS. Lithium effects on stem cells - advances in stem cell application in clinical medicine. Adv Cell Sci Tissue Cul

2018;2(1):14-24.

estimated 5.5 million Americans living with Alzheimer's dementia in 2017, an estimated 5.3 million are age 65 and older and approximately 200,000 individuals are under age 65 and have younger-onset Alzheimer's. Collectively the calculated cost in terms of health care dollars is \$259 billion. The projected increases for new cases of Alzheimer's disease are expected to reach 16 million by the year 2050 . One in three senior citizens in the country die as the result of Alzheimer's disease and a new case is diagnosed every 30 seconds in the United States [48].

$\mathrm{AD}$ is characterized by a buildup of amyloid plaques, caused by an accumulation of the amyloid-beta peptide [47]. In the brain of Alzheimer's patients amyloid plaques block the synapses between neurons. This results in the disruption of normal cell-to-cell signaling. The amyloid-beta protein activates GSK-3 $\beta$, resulting in the hyper phosphorylation of Tau proteins in neurons $[47,48]$. GSK-3 $\beta$ is the primary enzyme that contributes to the hyper phosphorylation of Tau proteins. The consequence of such abnormal phosphorylation is tangles of Tau proteins, referred to as "neurofibrillary tangles." Tau proteins are typically beneficial to the brain, aiding in the circulation of nutrients within brain cells, but neurofibrillary tangles disrupt this circulation and result in neuronal cell death $[47,48]$. Due to the important role GSK-3 $\beta$ plays in the progression of $\mathrm{AD}$, one of the most promising potential uses of lithium and its inhibitory effect on GSK-3 $\beta$ is the prevention of AD. Many clinical and experimental trials on humans and animals alike have proved the effectiveness of lithium on dementia and $\mathrm{AD}$, especially when treatment begins before the disease damages neurons beyond their point of repair [47]. Lithium's ability to reduce Tau hyper phosphorylation and the formation of $\mathrm{A} \beta$ plaques stands as one of the primary reasons why the drug serves as a great candidate for use in possible prevention and treatment of AD. A reduction in levels of Tau protein was indeed observed in a 2005 study on transgenic mice overexpressing mutant human Tau, a mutation that causes the development of neurofibrillary tangles.

As has been stated previously lithium exposure influences the production and action of GSK3 $\beta$ activity primarily by inhibiting its overall action. The next question is to understand whether lithium action via GSK3 $\beta$ activity influences in any way the mechanism by which lithium induces MSC proliferation. Mechanistic studies revealed that the ability of lithium to influence MSCs is dependent upon the activation of the GSK3 $\beta$ mediated via the WNT pathway. Lithium induced Ser9 phosphorylation, which results in the inhibition of GSK3 $\beta$ activity, $\beta$-catenin accumulation and WNT pathway activation. Utilizing a specific GSK-3 $\beta$ inhibitor SB216763 or siRNAmediated inhibition of GSK $3 \beta$ produced effects similar to those induced by lithium. In contrast, either quercetin, an inhibitor of the $\beta$-catenin/TCF pathway, or siRNA mediated knockdown of $\beta$-catenin abolished the proliferative effect of lithium, suggesting that lithium stimulates MSC proliferation via the GSK $3 \beta$ dependent $\beta$-catenin/WNT pathway. Collectively, these studies elucidate a novel role of lithium, which may not only provide a simple and effective way to strengthen MSC transplantation therapy efficacy, but also shed light on lithium's clinical application for the treatment of certain disorders resulting from $\beta$-catenin/WNT pathway suppression (Figure 2).
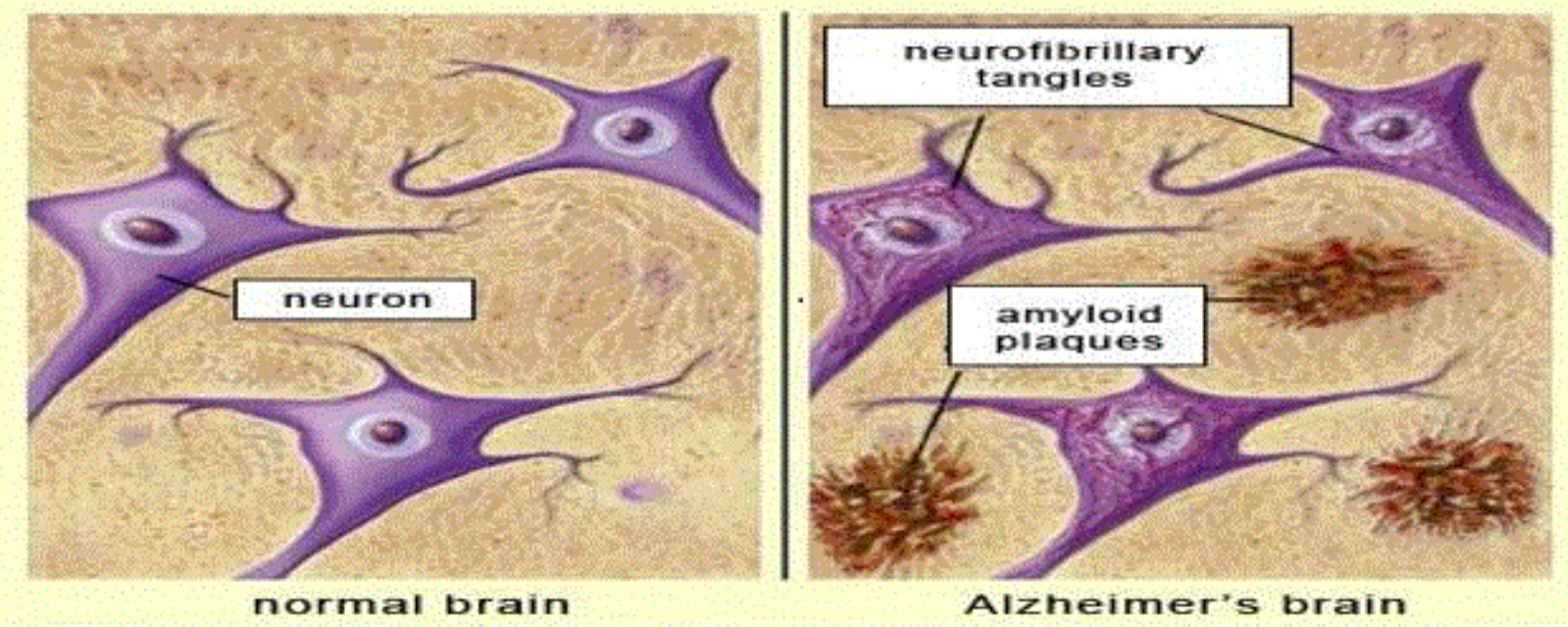

Figure 2: Reports have shown lithium therapy can prevent formation of amyloid beta plaques and neurofibrillary tangles composed of hyperphosphorylated tau proteins [56].

In addition to the ability of lithium to influence MSC proliferation, recently it has been reported to be effective inducing MSC differentiation to become neural cells [56]. Lithium possesses the ability to promote proliferation of green fluorescent protein (GFP)-MSCs in a dose dependent manner as verified by growth curve and bromodeoxyuridine (BrdU) incorporation assays. When these cells were co-cultured in neural induction medium, lithium $(0.1 \mathrm{mM})$ promoted neural differentiation of GFP-MSCs as verified by immunostaining and quantitative analysis. After transplantation of GFP-MSCs into the rat spinal cord, lithium treatment enhanced cell survival and neural differentiation after transplantation as verified by immunohistochemistry. These results open the door suggesting lithium could be a potential drug to augment the therapeutic efficiency of MSCs transplantation therapy in central nervous system (CNS) disorders. These effects could 
definitely enhance the field of clinical neuronal MSC transplantation.

\section{Lithium and Neural Stem Cells}

The effect of lithium on brain and blood function has been previously reviewed [57]. Lithium has been demonstrated to increase neurogenesis in the dentate gyrus of rodent hippocampus in addition to influencing the proliferation and differentiation of rat neural progenitor cells in hippocampus both in vitro and in vivo [58]. Lithium chloride (1-3 $\mathrm{mm}$ ) produced a significant increase in the number of bromodeoxyuridine (BrdU)-positive cells in high density cultures, but did not increase clonal size in low density cultures. Lithium chloride at $1 \mathrm{~mm}$, which is within the therapeutic range used clinically, also increased the number of cells double labeled with BrdU antibody and TuJ1 (a class III $\beta$-tubulin antibody) in high density cultures and the number of TuJ1-positive cells in a clone of low density cultures. While concurrently it decreased the number of glial fibrillary acidic protein positive cells in both cultures. These results suggest that lithium selectively increased differentiation of neuronal progenitors. The action of lithium appeared to enhance a neuronal subtype, calbindin D28k-positive cells, and to also involve a phosphorylated extracellular signal-regulated kinase and phosphorylated cyclic AMP response element binding protein-dependent pathway both in vitro and in vivo. These findings suggest lithium when tested at therapeutic concentrations may elicit its beneficial effects via facilitation of neural progenitor differentiation toward cells that demonstrate positive neuronal cell a phenotype.

As was just mentioned lithium influences stem cells. The importance of stem cells in $\mathrm{AD}$ therapy is becoming increasingly recognized. Lithium therapy promotes endogenous stem cell development, making lithium a very useful drug for neurogenesis. The types of stem cells most commonly emphasized in $\mathrm{AD}$ research are embryonic stem cells (ESCs), mesenchymal stem cells (MSCs), brain-derived neural stem cells (NSCs), induced pluripotent stem cells (iPSCs), and now very small embryonic stem cells (VSELs) $[59,60]$. A systematic review published in 2015 by FerensztajnRochowiak and Rybakowski evaluated the effects of lithium on many different classes of stem cells. In this review, it was reported that lithium has the ability to maintain the pluripotency of MSCs, while concentrations less than 5 $\mathrm{mmol} / \mathrm{L}$ promote MSC proliferation [61]. This is important due to the many roles of MSCs in the pathology of $\mathrm{AD}$; rodent models of $\mathrm{AD}$ have shown that MSC transplantation can improve spatial learning and memory deficits, inhibit cell death from accumulation of $A \beta$ protein and Tau, reduce the formation of $\mathrm{A} \beta$ plaques, reduce levels of inflammatory cytokines from activated microglia, and promote neurogenesis, synaptogenesis, and neuronal differentiation [59].

As demonstrated in rodent models, NSCs have also been shown to have a significantly positive impact on AD through the promotion of neurogenesis and cognitive function [60]. Lithium has been found to stimulate the self-renewal of neural progenitor cells, which serve as one of the two main sources of neurogenesis in the brain [61]. The drug is also reported to enhance the mitotic activity of Schwann cells and cause an increase in the presence BrdU labeled cells, used to detect proliferation of cells in brain tissues [60]. iPSCs have also shown great potential for use in AD treatment. When iPSCderived cholinergic neural precursors were transplanted into the hippocampus of a transgenic AD mouse model, these cells differentiated into mature cholinergic neurons and reversed spatial memory impairment. Additionally, iPSCs have been found to reduce the production of pro-inflammatory factors, improving neurological function in rodent models of ischemic stroke [62].

Ferensztajn-Rochowiak [61] suggest that increased mobilization of VSELs, increased mRNA expression of pluripotency and glial cell markers, and elevation of inflammatory cytokines are all associated with repeated exposures to stress and the specific chronic inflammation that occurs during BD pathogenesis. Long-term lithium treatment was shown to have a significant protective effect on all of these factors. The neuroinflammation processes involved in BD and $\mathrm{AD}$ are very similar, both strongly associated with microglial activation. For this reason, lithium can be presumed to have the same effect on AD pathology. Therefore, lithium's ability to decrease levels of VSELs, pluripotency and glial cell markers, and inflammatory cytokines contributes to lithium's candidacy as a treatment method for $\mathrm{AD}$ [62].

A study conducted by Ferensztajn-Rochowiak [60] to be published in 2018, evaluated the effects of long-term lithium treatment on concentrations of very-small embryonic-like stem cells (VSELs) and mRNA expression of pluripotency and glial markers in peripheral blood in patients diagnosed with bipolar disorder (BD). Participants included 30 BD patients in remission and 15 healthy control subjects. Of the $30 \mathrm{BD}$ patients, 15 had been treated continuously with lithium carbonate, with a mean treatment length of 16 years and serum concentration of $0.5-0.8 \mathrm{mmol} / \mathrm{L}$. Results showed that $\mathrm{BD}$ patients not taking lithium showed a significantly increased level of VSELs as compared to controls, while BD patients taking lithium showed a level of VSELs similar to controls. The number of VSELs in lithium treated BD patients was negatively correlated with the duration of lithium treatment and serum lithium concentration. In addition, among BD patients not treated with lithium, the mRNA levels of pluripotency markers, glial markers, and inflammatory cytokines were significantly higher than those of healthy controls [63]. This is an exciting development that will not only improve our understanding of bipolar depression, but also importantly how lithium can ameliorate the adverse effects associated with this mental disorder.

\section{Summary}

For more than sixty years lithium has been effectively used to treat patients with bipolar disorder and manic depression. Over these years many studies have demonstrated lithium has profound effects on both brain and blood in addition to its effective use in clinical psychiatry to treat mania and depression. For many years, the mechanisms of these effects of 
Citation: Gallicchio VS. Lithium effects on stem cells - advances in stem cell application in clinical medicine. Adv Cell Sci Tissue Cul 2018;2(1):14-24.

lithium were not known. Lithium is one of the lightest elements in the periodic table, an alkali metal that is in the same family as sodium and potassium. Why would lithium have any effect on manic depression? In the past two decades, several very interesting clues emerged, clinicians noted patients on lithium therapy often had increased white blood cell counts, a granulocytosis and reduced blood lymphocyte counts, a lymphopenia. Effects mediated by the ability of lithium to influence stem cells both in number and to influence their differentiation pathway. Following use of chemotherapy and radiation treatments in animals reduced white blood cell counts. Armed with the knowledge of the lithium therapy effect, veterinarians began treating animals with lithium. Lithium restored white blood cells and immune function faster. These side effects of lithium led to a better understanding of its mechanisms of action.

Scientists began applying lithium to bone marrow stem cells to see why it stimulated greater white blood cell production. These studies revealed that lithium affects phosphorylated inositides, a major cellular messenger system. Further studies showed that lithium stimulates and inhibits several phosphokinases and phosphatases, that all seemed to converge to inhibit a crucial enzyme called glycogen synthetase kinase $3-\beta$ (GSK3 $\beta$ ). This enzyme ostensibly phosphorylates and inhibits an enzyme called glycogen synthetase, which converts glucose to glycogen. But, more importantly, GSK3 $\beta$ phosphorylates several nuclear factors, including the nuclear factor of activated T cells (NFAT) and WNT/ $\beta$-catenin. Under the influence of lithium it became clear that the modulation of GSK3 $\beta$ activation/suppression mediated via $\mathrm{WNT} / \beta$-catenin is the key controller of many cellular programs that control growth, protection, and differentiation of stem cells. As the therapeutic efficacy of lithium continues to be tested clinically in an expanding spectrum of human diseases, the possibility that lithium will be demonstrated to be an effective therapeutic modality for these patients' only increases, providing patients with a possible therapeutic option that previously was not available. The fact that there is growing evidence that lithium influences a variety of stem cells only amplifies the optimism knowing this long standing drug may be used in the not too near distant future to determine its efficacy in a variety of human clinical disorders.

\section{References}

1. Gallicchio VS. Use of Trace Elements and Halotherapy in the Treatment of Human Diseases. In: Pharmacology and Nutritional Intervention in the Treatment of Disease 2014;43-96.

2. Chassarda BC, Galle P, Escaig F, et al. Bioaccumulation of lithium by marine organisms in European, American, and Asian coastal zones: microanalytic study using secondary ion emission. Accounts Acad Sci1984;299(18):719-24.

3. Schrauzer GN. Lithium: Occurrence, dietary intakes, and nutritional essentiality. J Am Coll Nutr 2002;21(1):14-21.

4. Kean S. The Disappearing Spoon. 2011.
5. Yacobi S, Ornoy A. Is lithium a real teratogen? What can we conclude from the prospective versus retrospective studies?. Isr J Psychiatry Relat Sci 2008;45(2):95-106.

6. Gallicchio VS, Chen MG. Modulation of murine pluripotential stem cell proliferation in vivo with lithium carbonate. Blood 1980;56(6):1150-52.

7. Gallicchio VS. Lithium and the Blood. Kargar 1991;1-150.

8. Abundance and form of the most abundant elements in Earth's continental crust. (http://www.gly.uga.edu/railsback/ Fundamentals/ElementalAbundanceTableP.pdf).

9. Cade JFJ. Lithium salts in the treatment of psychotic excitement. Med J Aust 1949;2(10):349-52.

10. Wang ZF, Fessler EB, Chuang DM. Beneficial effects of mood stabilizers lithium, valproate, and lamotrigine in experimental stroke models. Acta Pharmacol Sin 2011;32(12):1433-45.

11. Nahman S, Belmaker RH, Azab AN. Effects of lithium on lipopolysaccharide-induced inflammation in rat primary glia cells. Innate Immun 2012;18(3):447-58.

12. Matsebatlela TM, Mogodiri RK, Hart DA, et al. Resistance to lithium-induced apoptosis in a lithium tolerant clone of HL-60 promyelocytes. J Trace Microprobe Techn 2000;18:163-70.

13. Becker RW, Tyobeka EM. Lithium enhances proliferation of the HL-60 promyelocytic leukemia cells. Leuk Res 1990;14(10):879-84.

14. LMadiehe AM, Mampuru LJ, Tyobeka EM. Induction of 7:99 apoptosis in HL-60 by lithium. Biochem Biophys Res Commun 1995; 209:768-74.

15. Mampuru LJ, Abotsi EK, Wachira SJM. Calyculin-A potentiates growth and apoptotic effects of lithium in HL-60 cells. J Trace Microprobe Tech 1999;17:367-78.

16. Shai LJ, Abotsi EK, Tyobeka EM, et al. Partial involvement of c-jun proto-oncogene in growth enhancing and cytotoxic effects of lithium in vitro. Cell Mol Biol 2003; 49(7): 1137-43.

17. Klein PS, Melton DA. A molecular mechanism for the effect of lithium on development. Proc Natl Acad Sci 1996;93(16):8455-59.

18. Stambolic V, Ruel L, Woodgett JR. Lithium inhibits glycogen synthase kinase-3 activity and mimics wingless signaling in intact cells. Curr Biol 1996;6(12):1664-68.

19. Jope RS. Lithium and GSK-3: one inhibitor, two inhibitory actions, multiple outcomes. Trends Pharmacol Sci 2003; 24(9):433-41.

20. Chiu CT, Chuang DM. Molecular actions and therapeutic potential of lithium in preclinical and clinical studies of CNS disorders. Pharmacol Ther 2010;128(2):281-304.

21. Ryves WJ, Harwood AJ. Lithium inhibits glycogen synthase kinase-3 by competition for magnesium. Biochem Biophys Res Commun 2001;280(3):715-20.

22. Chuang DM, Wang Z, Chiu CT. GSK-3 as a target for lithium-induced neuroprotection against excitotoxicity in neuronal cultures and animal models of ischemic stroke. Front Mol Neurosci 2011;4:15. 
23. Ozaki N, Chuang DM. Lithium increases transcription factor binding to AP-1 and cyclic AMP- responsive element in cultured neurons and rat brain. $J$ Neurochem 1997; 69(6)2336-44.

24. Chen RW, Qin ZH, Ren M, et al. Regulation of c-Jun Nterminal kinase, p38 kinase and AP-1 DNA binding in cultured brain neurons: roles in glutamate excitotoxicity and lithium neuroprotection. J Neurochem 2003;84(3): 566-75.

25. Wang MJ, Huang YH, Chen WF, et al. Glycogen synthase kinase-3beta inactivation inhibits tumor necrosis factor alpha production in microglia by modulating nuclear factor kappa $\hat{A}$ and MLK3/JNK signaling cascades. J Neuroinflammation 2010;7:99-117.

26. Huang WC, Lin YS, Wang CY, et al. Glycogen synthase kinase-3 negatively regulates anti- inflammatory interleukin-10 for lipopolysaccharide-induced iNOS/NO biosynthesis and RANTES production in microglial cells. Immunol 2009;128:275-86.

27. Jeso BD, Ulianich L, Racioppi I, et al. Serum withdrawal induces apoptotic cell death in KI-RAS transformed but not in normal differentiated thyroid cells. Biochem Biophys Res Commun 1995;214(3):819-24.

28. Becker RW, Gallicchio VS. Mitogenic effects of lithium on the hematopoietic system. J Trace Microprobe Techn 1996;14(10):799-812.

29. Focosi D, Azzara A, Kast RE, et al. Lithium and hematology: established and proposed uses. J Leuko Biol 2008;85(1):20-8.

30. Becker RW, Gallicchio VS. Modulation by lithium of the formed elements of blood. Proceedings of the international symposium on trace elements in humans 1997;525-47.

31. Lucas KC, Hart DA, Becker RW. Porcine proximal tubular cells (LLC-PK1) are able to tolerate high levels of lithium chloride in vitro: assessment of the influence of 1-20 mM $\mathrm{LiCl}$ on cell death and alterations in cell biology and biochemistry. Cell Biol Int 2010;34(2):225-33.

32. Chin L, Artandi SE, Shen Q, et al. P53 deficiency rescues the adverse effects .of telomere loss and cooperates with telomere dysfunction to accelerate carcinogenesis. Cell 1999;97(4):527-38.

33. Scholtz W. IL-6 in disease: cause or cure? Immunopathology 1996; 3:131-50.

34. Parry SL, Sebbag M, Feldman M, et al. Contact with T cells modulates monocytes IL-10 production. J Immunol 1997;158(8):3673-81.

35. Hammond WP, Dale DC. Lithium Therapy of Canine Cyclic Hematopoiesis. Blood 1980;55(1):26-8.

36. Leclerc A, Abrams-Ogg AC, Kruth SA, et al. Effects of lithium carbonate on carboplatin-induced thrombocytopenia in dogs. Amer J Vet Res 2010;17(5): 555-63.

37. Anthony CC, Abrams OA. The use of lithium carbonate to prevent lomustine-induced myelosuppression in dogs. Can J Vet Res 2010;75(1):73-6.
38. Sontas HB, Dokuzeylu B, Turna O, et al. Estrogen-Induced Myelotoxicity in Dogs: A Review. Can Vet J 2009;50(10): 1054-58.

39. Conrado DO, Rodrigues DA, Lacerda DA, et al. Use of lithium carbonate in the treatment of a suspected case of oestrogen-induced bone marrow aplasia in a bitch. Vet Res 2009;164(9):274-85.

40. Schmidt MM, Guan K, Wobus A. Lithium influences differentiation and tissue-specific gene expression of mouse embryonic stem (ES) cells in vitro. Int $\mathrm{J}$ Dev Biol 2001;45(2):421-29.

41. Qureshi WM, Latiff ML, Parket TL, et al. Lithium carbonate teratogenic effects in chick cardiomyocyte micromass system and mouse embryonic stem cell derived cardiomyocyte. Possible protective role of myo-inositol phosphate. Reprod Toxicol 2014;46:106-14.

42. Bach RO, Gallicchio VS. Lithium and Cell Physiology. 1990, Spiger-Verlag.

43. Birch NJ. Lithium and the Cell: Pharmacology and Biochemistry. 1991, Academic Press, London.

44. Birch NJ, Padgham C, Hughes MS. Lithium in Medicine and Biology. 1993, Marius Press, London.

45. Birch NJ. Inorganic Pharmacology of the Lithium Ion. Chem Rev 1999;99(9):2659-82.

46. Quiroz JA, Gould TD, Manji MK. Molecular Effect of Lithium. Mol Interv 2004;4:(5)259-72.

47. Bauer M, Grof P, Muller O B. Lithium in Neuropsychiatry: The Comprehensive Guide. 2006, Informa Health Care, Berlin.

48. Atroshi F. Pharmacology and Nutritional Intervention in the Treatment of Disease. 2014, Intech, Croatia, London.

49. Gallicchio VS, Messino MJ, Hulette BC, et al. Lithium and hematopoiesis: Effective experimental use of lithium as an agent to improve bone marrow transplantation. J Med 1992;23(3\&4):195-216.

50. Gallicchio VS, Hughes NK. Lithium stimulation of hematopoiesis: demonstration that lithium influences the spatial distribution of hematopoietic progenitor cells within endosteal bone marrow. Lithium 1992; 3:117-24.

51. Huang J, Zhang Y, Berenev A, et al. Pivotal role for glycogen synthase kinase-3 in hematopoietic stem cell homeostasis in mice. J Clin Invest 2009;119(12):3519-29.

52. Wang Q, Xu X, Li J, et al. Lithium, an anti-psychotic drug, greatly enhances the generation of induced pluripotent stem cells. Cell Res 2011;21:1424-35.

53. Chen HM, DeLong CJ, Bame M, et al. Transcripts involved calcium signaling and telencephalic neuronal fate are altered in induced pluripotential stem cells from bipolar disorder patients. Translational Psychiatry 2014;4:1-8.

54. Zhu ZI, Yin J, Guan J, et al. Lithium stimulates human bone marrow derived mesenchymal stem cell proliferation through GSK-3 $\beta$-dependent $\beta$--catenin/Wnt pathway activation. FEBS J 2014;281(23):5371-89.

55. Duvall A, Gallicchio VS. Lithium Treatment in Clinical Medicine: History, Current Status and Future Use. J Cell Sci Ther 2017; 8: 270. 
Citation: Gallicchio VS. Lithium effects on stem cells - advances in stem cell application in clinical medicine. Adv Cell Sci Tissue Cul 2018;2(1):14-24.

56. Bao TD, Guan JT, Ya XH, et al. Lithium enhanced cell proliferation and differentiation of mesenchymal stem cells to neural cells in rat spinal cord. Int J Clin Exp Pathol 2015; 8(3): 2473-83

57. Young W. Review of lithium effects on the brain and blood. Cell Transplantation 2009;18(9):951-75.

58. Seuk KJ, Chang MY, In $\mathrm{T}$, et al. Lithium selectively increases neuronal differentiation of hippocampal neural progenitor cells both in vitro and in vivo. J Neurochem 2004; 89(2):324-36.

59. Duncan T, Valenzuela M. Alzheimer's disease, dementia, and stem cell therapy. Stem Cell Res Ther 2017;8(1):111.

60. Ferensztajn-Rochowiak E, Rybakowski JK. The effect of lithium on hematopoietic, mesenchymal, and neural stem cells. Pharmacol Rep 2016;68(2):224-30.

61. Ferensztajn-Rochowiak E, Kucharska-Mazur J, Tarnowski $\mathrm{M}$, et al. Stem cells, pluripotency and glial markers in peripheral blood of bipolar patients on long term lithium treatment. Progress in Neuropsychopharmacology \& Biological Psychiatr 2018;80:28-33.

62. Zhu Z, Yin J, Guan J, et al. Lithium stimulates human bone marrow derived mesenchymal stem cell proliferation through GSK-3 $\beta$-dependent b-catenin/Wnt pathway activation. FEBS J 2014; 281(23): 5371-89.

63. Forlenza OV, Diniz BS, Radanovic M, et al. Diseasemodifying properties of long-term lithium treatment for amnestic mild cognitive impairment: randomised control trial. British J Psychiatry 2011:198(5); 351-6.

\section{*Correspondence to:}

Vincent S Gallicchio

Departments of Biological Sciences, Microbiology, and Public Health Sciences

College of Science and College of Health and Human Development

Clemson University

Clemson

South Carolina

USA

E mail: vsgall@clemson.edu 